\title{
Estimation of a Safe Level for Occupational Exposure to Vinyl Chloride Using a Benchmark Dose Method in Central China
}

\author{
Jie JiaO ${ }^{1,2 \#}$, Nan-nan FenG ${ }^{1 \#}$, Yong $\mathrm{LI}^{1 \#}$, Yuan $\mathrm{SuN}^{1}$, Wu YaO ${ }^{3}$, Wei WANG ${ }^{3}$, \\ Guang-hui ZHANG ${ }^{1}$, Shi-yi Sun ${ }^{4}$, Hong-shan TAN ${ }^{1}$, Qi WANG ${ }^{1}$, Yiliang ZHU ${ }^{5}$, \\ Yongliang $\mathrm{LI}^{6}$, Paul W. Brandt-RAUF ${ }^{6}$ and Zhao-lin XIA ${ }^{1}$ \\ ${ }^{1}$ Department of Occupational Health and Toxicology, School of Public Health, Fudan University, China, ${ }^{2}$ Henan \\ Institute of Occupational Health, China, ${ }^{3}$ Department of Occupational Health, School of Public Health, Zhengzhou \\ University, China, ${ }^{4}$ Institute of Occupational Medicine, China, ${ }^{5}$ Department of Epidemiology and Biostatistics, \\ College of Public Health, University of South Florida, USA and ${ }^{6}$ School of Public Health, University of Illinois at \\ Chicago, USA
}

\begin{abstract}
Estimation of a Safe Level for Occupational Exposure to Vinyl Chloride Using a Benchmark Dose Method in Central China: Jie JIAO, et al. Department of Occupational Health and Toxicology, School of Public Health, Fudan University, ChinaObjectives: The aim of this study was to estimate a benchmark dose (BMD) for chromosome damage induced by vinyl chloride monomer (VCM) in VCMexposed workers in central China and validate the published results in Shanghai. Methods: VCMexposed workers who had been exposed to VCM for at least one year $(n=463)$ and matched subjects not exposed to VCM or other toxins $(n=273)$ were asked to participate in this study. Micronucleus (MN) frequency based on the cytokinesis-block micronucleus assay (CBMN) was used as a biomarker for chromosome damage induced by VCM exposure. Results: The MN frequency in the VCM-exposed workers was significantly higher than that in the control group, and multivariate Poisson regression suggested that gender, smoking status and VCM exposure were the significant factors influencing the risk of increased MN frequency. When subjects were further stratified according to gender and smoking status, the results showed that female VCMexposed workers were more susceptible than the males to the risk of increased $\mathrm{MN}$ frequency. The $\mathrm{MN}$ frequency of smokers was significantly higher than that of nonsmokers in the control group. Our study also suggested that there was a strong dose-response rela-
\end{abstract}

Received Jul 26, 2011; Accepted Mar 27, 2012

Published online in J-STAGE May 29, 2012

Correspondence to: Z.-l. Xia, Department of Occupational Health and Toxicology, School of Public Health, Fudan University, 138 Yixueyuan Road, Shanghai, 200032, China (e-mail: zlxia@shmu. edu.cn)

${ }^{\#}$ These authors contributed equally to this work. tionship between VCM CED and the increased risk of MN frequency in the total group, males and females. The BMDL ${ }_{10}$ was found to be $630.6,670.2$ and $273.7 \mathrm{mg}$ year for all VCM-exposed workers, males and females, respectively. Conclusions: These results invite further scrutiny of the current VCM occupational exposure limits and warrant further study of the risk of VCM genotoxicity and carcinogenicity.

(J Occup Health 2012; 54: 263-270)

Key words: Benchmark dose, Chromosome damage, Micronucleus frequency, Vinyl chloride monomer

Vinyl chloride monomer (VCM) is a known cause of angiosarcoma of the liver ${ }^{1)}$. The International Agency for Research on Cancer (IARC) classified VCM as a confirmed human carcinogen in 1974, 1978 and $1987^{2}$. VCM is extensively used in the production of vinyl chloride polymer, copolymer resin, packaging materials and wire and cable coatings as well as in industrial and laboratory intermediates ${ }^{3)}$. The wide human exposure to this compound in different industries throughout the world causes great concern for human health. Previous studies, 5) suggested that the two oxidative metabolites of VCM (chloroethylene oxide (CEO) and chloroacetaldehyde (CAA)) could produce mutagenic effects. In addition to mutations, other genotoxic effects were reported such as gene conversion, sister chromatid exchanges (SCE), micronuclei $(\mathrm{MN})$, mitotic recombination, chromosomal aberrations (CA) and cell transformation ${ }^{3,6,7)}$. The MN assay has become a well-known and promising indicator to investigate the genotoxicity of a variety of chemicals especially using the cytokinesis-block micronucleus $(\mathrm{CBMN})$ assay $^{8,9}$. The CBMN method 
is the most reliable method for measuring $\mathrm{MN}$ in peripheral blood lymphocytes (PBL) $)^{9}$. In this assay, $\mathrm{PBL}$ are induced to divide ex vivo in culture, and $\mathrm{MN}$ are scored in cells that have completed one nuclear division; the latter are recognized as binucleated cells after inhibition of cytokinesis using cytochalasin-B. The CBMN method can assess chromosome damage including chromosome breakage and chromosome loss reliably in occupational and environmental exposures $^{7,10-16)}$. Some investigations ${ }^{7,17)}$ have demonstrated that the frequency of $\mathrm{MN}$ is increased in lymphocytes of individuals occupationally exposed to VCM.

The benchmark dose (BMD) was defined by Crump $^{18)}$ as a statistical lower confidence limit corresponding to a moderate increase in risk (1-10\%) above the background risk. Crump suggested that the BMD could be used to replace the no observed adverse effect level (NOAEL) or the lowest observed adverse effect level (LOAEL) for noncarcinogenic effects in the regulatory process for setting acceptable and safe exposure levels of toxic compounds ${ }^{19,20)}$. The main advantage with the BMD methodology is that it uses all dose-response data from a study ${ }^{21}$. Gaylor et $a .^{22)}$ redefined the BMD as the point estimate of the dose corresponding to a specified low level of risk and suggested that the concept of BMDL (lower confidence limit of benchmark dose) could be used as a replacement for the NOAEL or LOAEL ${ }^{20)}$. The BMDL resulting from this approach is more accurate than a NOAEL or LOAEL. The BMDL is typically calculated using the lower 95\% confidence limit on the dose-response curve to a 1-10\% level of risk above the background. A $10 \%$ benchmark response level (BMR) is conventionally used for dichotomous end points because it is at the low end of the observable range for many common study designs. Various investigations ${ }^{23-30)}$ have performed risk assessments of the non-cancer effects of toxic exposures using the benchmark dose (BMD) method. A previous study ${ }^{31)}$ evaluated the effects of occupational exposure to vinyl chloride monomer (VCM) on the micronucleus and estimated a benchmark dose for chromosome damage induced by VCM in VCM-exposed workers. The objective of the present study was conducted to confirm the finding in the previous study that there was the strong relationship between VCM CED and chromosome damage using the BMD approach; likewise, $\mathrm{CBMN}$ was used as an indicator of chromosome damage induced by VCM.

\section{Materials and Methods}

\section{Study population}

Workers from a VC polymerization plant in Henan Province, central China, were recruited during routine medical evaluations. After informed consent was obtained, workers underwent an interviewer-administered questionnaire including data on age, lifestyle, professional and medical history and smoking and drinking habits. Eligibility was based on employment records and was defined as occupational exposure to VCM for at least $1 \mathrm{yr}$. Workers occupationally exposed to VCM who had completed questionnaires and finished the CBMN assay were included in the VCM exposure group (463 in total; 268 males and 195 females, $32.5 \pm 8.81 \mathrm{yr})$. For the control group, we selected 273 (137 males and 136 females, $25.5 \pm 7.26 \mathrm{yr})$ subjects working in the same city as the VCM exposure group and without exposure to VCM and other toxic chemicals as non-occupationally exposed (reference) subjects and constituted the control group. Although the age variable is not comparable between VCM exposure group and the control group, it was adjusted when the authors performed the statistical analysis.

\section{VCM exposure assessment}

VCM air samples were collected at different work sites of the plant and analyzed by gas chromatography to determine VCM air concentrations for the various work sites and estimate the cumulative exposure dose for each worker. Since the cumulative exposure is related to work location, work duration and many other factors, we can use the same method as described ${ }^{17,32)}$ previously to assess the cumulative exposure dose of VCM from the VCM air concentrations. In brief, the following equation was used to calculate cumulative exposure dose: cumulative exposure dose $(\mathrm{mg})=\sum(\mathrm{C} \times \mathrm{M} \times \mathrm{T}) \times \mathrm{A} \times 70 \% / 10^{6}$, where $\mathrm{C}\left(\mathrm{mg} / \mathrm{m}^{3}\right)$ was the geometric mean of the VCM exposure concentration for each month in a special workplace, calculated for all different work sites; $M$ was the number of exposure months of each year for a VCM worker; $\mathrm{T}$ was the $2 \mathrm{~h}$ exposure time in each working day, 20 days in each month, giving 2,400 min of exposure time per month; and A was the alveolar ventilation (male average, $6,500 \mathrm{ml} / \mathrm{min}$; female average, $4,300 \mathrm{ml} / \mathrm{min}$; $30 \%$ dead space).

For the control group, the VCM exposure dose was evaluated according to the published literature ${ }^{33)}$. In brief, general environmental levels of $\mathrm{VC}$ from all sources are likely to lead to average exposures of $2-10 \mu \mathrm{g} /$ day. Smokers may inhale additionally up to $0.5 \mu \mathrm{g} / \mathrm{day}$. Exposures from food and water are less than $0.1 \mu \mathrm{g} / \mathrm{day}$, so these exposures were not included when evaluating the total cumulative exposure dose of VCM in the present study.

\section{CBMN assay}

The CBMN assay was performed according to a standard protocol as described by Fenech ${ }^{34)}$. In 
brief, $0.5 \mathrm{ml}$ heparin-anticoagulated whole blood was collected, transferred to $4.5 \mathrm{~m} l$ RPMI 1640 medium and incubated at $37^{\circ} \mathrm{C}$ in $5 \% \mathrm{CO}_{2}$ for $44 \mathrm{~h}$, and cytokinesis was blocked with $6 \mu \mathrm{g} / \mathrm{m} l$ cytochalasin-B (Sigma-Aldrich, St.Louis, MO, USA). Twenty-eight hours after the addition of cytochalasin-B, cells were harvested and fixed with methanol and acetic acid at a ratio of 3:1. Slides were air-dried, stained with Giemsa and scored for CBMN. For each subject, 1,000 binucleated lymphocytes with well-preserved cytoplasm were scored blindly.

\section{BMD method}

In the present study, VCM cumulative exposure dose was used as the dose parameter. We used the BMDL to estimate the lower confidence limit of the population critical concentration of the cumulative exposure dose of VCM-exposed workers.

In this study, the analysis of regression and curve estimation were performed using the Benchmark Dose Software (Version 2.1.2) from the US Environmental Protection Agency (EPA). A BMR of $10 \%$ was used in calculating the BMD. This value is consistent with $10 \%$ of the BMR suggested by the US EPA (2003). The normal cut-off point was defined as being based on the $95 \%$ limit value in the control group. The BMD analysis for the total group was used as an example to explain the models selected. We fit the data to a Probit model, Logistic model and Log-logistic model, respectively (Table 5). $p$ values $\geq 0.05$ can be found in all models above mentioned. We chose the log-logistic model to analyze the data because the AIC value of this model was the smallest among the three models. The BMDL was specified as the lower $95 \%$ confidence limit of the dose corresponding to the BMR in the study, and the smooth option of the model was applied (figure not shown).

The equation of the log-logistic model is

\author{
$\mathrm{P}$ [chromosome damage $]=$ \\ $\mathrm{c}+(1-\mathrm{c}) /[1+\mathrm{EXP}(-\mathrm{a}-\mathrm{b} * \mathrm{LOG}(\mathrm{CED}))]$.
}

\section{Statistical analysis}

The multivariate Poisson regression model was performed to examine the relationship between the cumulative exposure dose and $\mathrm{MN}$ frequency. Potential confounding variables included in the final models were age (continuous variables), smoking status, drinking status, gender and VCM exposure status (continuous variables). The risk of chromosome damage was estimated by calculating frequency ratios (FR) (herein and in the following text, FR is the IRR (incidence rate ratio)) and $95 \%$ confidence intervals $(C I)$, with $F R=e^{\beta}(e \approx 2.71828)$. For categorical variables, the FR denotes the proportional increase of the micronucleus frequency in a comparison group relative to the reference group; for example, an FR of 1.48 for females versus males means a $48 \%$ increase in the micronucleus frequency in females. For continuous variables, the FR represents the proportional increase of micronucleus frequency due to the increase of one unit of the variable evaluated; for example, an FR for age of 1.11 means $11 \%$ increase in the micronucleus frequency per year of age. All $p$ values presented are 2-sided, and all analyses were performed using the SAS Version 9.2 Software (SAS Institute).

\section{Results}

\section{VCM exposure assessment}

We estimated that the exposure dose in the VCM exposure group ranged from $34.49 \mathrm{mg}$ to $113.06 \mathrm{mg}$ in air, with the median dose being $58.4 \mathrm{mg}$. The personal cumulative exposure dose in the VCM exposure group ranged from $886 \mathrm{mg}$ to $127,915 \mathrm{mg}$, with the median dose being $18,910 \mathrm{mg}$.

Based on the above data, we assessed the VCM cumulative exposure dose (CED) in mg-year. In the

Table 1. Poisson regression model for all factors of $\mathrm{MN}$

\begin{tabular}{cccrc}
\hline Parameter & Estimate & FR $(95 \% \mathrm{CI})$ & \multicolumn{1}{c}{$\mathrm{X}^{2}$} & \multicolumn{1}{c}{$p$} \\
\hline Intercept & -0.162 & - & 4.93 & 0.027 \\
Age & -0.059 & $0.94(0.82-1.08)$ & 0.72 & 0.396 \\
Smoke* & 0.173 & $1.19(1.01-1.40)$ & 4.11 & 0.043 \\
Drink & 0.121 & $1.13(0.97-1.32)$ & 2.37 & 0.123 \\
Gender** & -0.256 & $0.77(0.68-0.88)$ & 14.97 & 0.001 \\
VCM exposure** & 0.952 & $2.60(2.21-3.03)$ & 139.10 & $<0.001$ \\
\hline
\end{tabular}

MN: micronucleus; VCM: vinyl chloride; FR: frequency ratio of mean MN frequency between a comparison and a reference group, the results derived from Multivariate Poisson regression models when these impact factors were adjusted by each other.

*, **: $p<0.05 . p<0.01$ with regard to the corresponding group, respectively. 
Table 2. MN frequency of VCM exposure and control group by gender and smoking status

\begin{tabular}{|c|c|c|c|c|c|c|}
\hline & \multicolumn{3}{|c|}{ Control } & \multicolumn{3}{|c|}{ Exposure } \\
\hline & $\mathrm{N}$ & $\mathrm{MN}^{\mathrm{a}}$ & FR $(95 \%)$ & $\mathrm{N}$ & $\mathrm{MN}^{\mathrm{a}}$ & FR $(95 \%)$ \\
\hline \multicolumn{7}{|l|}{ Gender } \\
\hline Male & 137 & $0.70(0,5)$ & 1 & 268 & $1.85(0,10)$ & 1 \\
\hline Female & 136 & $0.88(0,5)$ & $0.80(0.61-1.05)$ & 195 & $2.17(0,22)$ & $1.17(1.03-1.33)^{*}$ \\
\hline \multicolumn{7}{|l|}{ Smoking } \\
\hline Non-smokers & 212 & $0.73(0,5)$ & 1 & 280 & $1.92(0,22)$ & 1 \\
\hline Smoker & 61 & $1.00(0,5)$ & $1.38(1.02-1.85)^{*}$ & 183 & $2.27(0,10)$ & $0.97(0.85-1.11)$ \\
\hline
\end{tabular}

MN: micronucleus; VCM: vinyl chloride; FR: frequency ratio of mean MN frequency between a comparison and a reference group, the results derived from Poisson regression models fitted separately to the control or exposure group with a single risk factor under consideration;

a: The numbers in parenthesis in MN column mean minimum and maximum MN frequency, respectively.

*: $p<0.05$ with regard to the corresponding group.

Table 3. Differences in $\mathrm{MN}^{*}$ frequency by CED* of $\mathrm{VCM} *$

\begin{tabular}{crcrcc}
\hline CED (mg-year) & \multicolumn{1}{c}{$\mathrm{N}$} & $\mathrm{MN}(\%)$ & $\chi^{2}$ & $p$ & FR* (95\%CI) \\
\hline $0-$ & 328 & $1.12(0.9)^{\mathrm{a}}$ & & & 1 \\
$167-$ & 111 & $1.40(0.10)$ & 2.02 & 0.1549 & $1.25(0.92-1.69)$ \\
$667-$ & 76 & $1.86(0.10)$ & 9.87 & 0.0001 & $1.66(1.21-2.27)$ \\
$1,667-$ & 222 & $2.13(0.22)$ & 32.46 & $<0.0001$ & $1.90(1.53-2.38)$ \\
\hline
\end{tabular}

*MN: micronucleus; *CED: cumulate exposure dose of vinyl chloride monomer; *VCM: vinyl chloride; *FR: frequency ratio of mean MN frequency between a comparison and a reference group, the results derived from Poisson regression models fitted separately to the control or exposure group with a single risk factor under consideration; ${ }^{\text {a: }}$ The values in parenthesis in $\mathrm{MN}$ mean minimum and maximum $\mathrm{MN}$ frequency, respectively.

subsequent analysis, as shown in Table 4 , four ordinal CED groups were used: $0<\mathrm{CED} \leq 167,167<\mathrm{CED} \leq 667$, $667<\mathrm{CED} \leq 1,667$ and $1,667<\mathrm{CED}$. The group CED levels were represented by the group median CED level.

$M N$ frequency and its association with risk factors

The mean and range of CBMN frequencies in the VCM-exposed group and control group were 1.99 and 0-22 and 0.79 and $0-4$, respectively. Herein and in the following text, the scale of $\mathrm{CBMN}$ frequencies concretely refer to per mille. $\mathrm{MN}$ frequency in the VCM-exposed workers was significantly higher than in the control group [FR (95\% CI): 2.52 (2.17-2.93)]. As shown in Table 1, multivariate Poisson regression suggested that gender, smoking status and VCM exposure were the significant factors influencing the risk of increased MN frequency. All the data exclusive of age and VCM exposure status (age and VCM exposure status are continuous data) were analyzed as binary data in the Poisson regression analysis.

Subjects were further stratified according to gender and smoking status. The results for FR derived from
Poisson regression analysis (Table 2) showed that among VCM-exposed workers, the MN frequency of females was significantly higher than that of males [FR (95\% CI): 1.17 (1.03-1.34)], but there was no similar association in the control group. The $\mathrm{MN}$ frequency of smokers was significantly higher than that of nonsmokers in the control group [FR $(95 \% \mathrm{CI}): 1.38$ (1.02-1.85)].

$M N$ frequency and its association with VCM exposure

In this study, as we mentioned above, we found that subjects occupationally exposed to VCM had a higher risk of having an increased $\mathrm{MN}$ frequency than the control group. VCM-exposed workers were further divided according to different CEDs (Table 3). The results derived from Poisson regression suggested that there was a strong dose-response relationship between VCM CED and the risk of increased MN frequency.

The values of BMD and BMDL of VCM cumulative exposure dose as chromosome damage indicators

We defined the normal cut-off point based on the 95\% limit value in the control group such that if a 
Table 4. Chi-square trend test for chromosome damage with VCM exposure

\begin{tabular}{ccccccc}
\hline \multirow{2}{*}{ CED* $^{*}$ (mg-year) $)$} & \multicolumn{2}{c}{ Combined } & \multicolumn{2}{c}{ Male } & \multicolumn{2}{c}{ Female } \\
\cline { 2 - 7 } & $+/-$ & $\%$ & $+/-$ & $\%$ & $+/-$ & $\%$ \\
\hline $0-$ & $36 / 292$ & 10.9 & $17 / 143$ & 10.6 & $19 / 149$ & 11.3 \\
$167-$ & $22 / 89$ & 19.8 & $12 / 48$ & 20.0 & $10 / 41$ & 19.6 \\
$667-$ & $18 / 58$ & 23.6 & $8 / 23$ & 25.8 & $10 / 35$ & 22.2 \\
$1,667-$ & $74 / 208$ & 33.3 & $49 / 106$ & 31.6 & $25 / 42$ & 37.3 \\
\hline Linear Trend & \multicolumn{2}{c}{$\mathrm{X}^{2}=23.56$} & \multicolumn{2}{c}{$\mathrm{X}^{2}=20.66$} & $\mathrm{X}^{2}=20.10$ \\
$p$ & \multicolumn{2}{c}{$<0.001$} & \multicolumn{2}{c}{$<0.001$} & $<0.001$ \\
\hline
\end{tabular}

chromosome damage was defined as micronucleus frequency $\geq 3 \%$; VCM: vinyl chloride monomer; CED: the median cumulate exposure dose to vinyl chloride in each CED group was used as the dose level in chi-square trend test. For example, the median CED were 3.8, 346.6, 1,134.2, 5,132.2 mg-year for both genders combined.

Table 5. The parameters of different models for BMD and BMDL when total was taken as an Indicator of chromosome damage

\begin{tabular}{clrccc}
\hline Indicator & Model & BMD & BMDL & AIC & $p$ value \\
\hline Total* & Probit & $1,125.8$ & 882.9 & 754.95 & 0.06 \\
Total & Logistic & $1,150.8$ & 914.9 & 755.10 & 0.06 \\
Total & Log-logistic & 913.3 & 630.6 & 753.74 & 0.11 \\
\hline
\end{tabular}

BMD: benchmark dose; BMDL was specified as the lower $95 \%$ confidence limit on the cumulate exposure dose of vinyl chloride corresponding to the benchmark dose reaction in this study; *Total: the subjects of genders combined.

Table 6. BMDL Estimates of CED of VCM (mg-year) for chromosome damage

\begin{tabular}{ccccccccc}
\hline Indicator & $\mathrm{N}$ & $\mathrm{a}$ & $\mathrm{b}$ & $\mathrm{c}$ & BMD-0.1 & BMDL-0.1 & AIC & $p$ values \\
\hline Total & 737 & -9.01 & 1 & 0.11 & 913.3 & 630.6 & 753.74 & 0.11 \\
Male & 406 & -13.63 & 1 & 0.22 & $1,420.6$ & 670.2 & 465.72 & 0.63 \\
Female & 331 & -8.26 & 1 & 0.11 & 431.4 & 273.7 & 301.92 & 0.57 \\
\hline
\end{tabular}

VCM: vinyl chloride monomer; Model: $\mathrm{p}=\mathrm{c}+(1-\mathrm{c}) /[1+\mathrm{EXP}(-\mathrm{a}-\mathrm{b} * \mathrm{LOG}(\mathrm{CED}))]$, here CED is the median cumulate exposure dose to vinyl chloride in each CED group was used as the dose level in chi-square trend test; $\mathrm{a}, \mathrm{b}$ and $\mathrm{c}$ are coefficients derived from the data; Excess risk at BMD is $0.10,{ }^{*} p$ values were obtained from chi-square test; use the Pearson goodness of fit test; if $p>0.05$, means the equation is succeed fit.

value was higher than this, we would consider the level of chromosome damage as abnormal (positive). In this study, chromosome damage was defined as an MN frequency $\geq 3 \%$ o.

To further confirm the dose-response between VCM exposure and chromosome damage as well as to facilitate benchmark dose computation, we conducted an alternative dose-response assessment by considering cases of chromosome damage defined by this cut-off of an $\mathrm{MN}$ frequency $\geq 3 \%$. As shown in Table 4, there was a significant dose-response relationship between VCM exposure in the total group, male group and female group and chromosome damage based on $\mathrm{MN}$ frequency.
The log-logistic model as implemented in BMDS permits only aggregated data. Thus we used group prevalence data of chromosome damage (Table 6), ignored individual risk factors and utilized the median VCM CED of each group as the "dose" for each individual in that group. Concretely, 3.8, 346.6, 1,134.2 and $5,132.2 \mathrm{mg}$-year were the medians of the four CED groups in total (gender combined); 3.9, 356.5, $1,273.4$ and $6,254.8 \mathrm{mg}$-year were the medians for males; and 3.7, 322.4, 1,052.5 and 3,848.7 mg-year were the medians for females.

The estimated parameters and corresponding values of $\mathrm{BMDL}_{10}$ are presented in Table 6. $p$ values provide a goodness-of-fit measure for the models. We found 
that $\mathrm{BMDL}_{10}$ values were $630.6 \mathrm{mg}$-year, $670.2 \mathrm{mg}$-year and $273.7 \mathrm{mg}$-year for total VCM-exposed workers, male VCM-exposed workers and female VCM-exposed workers, respectively.

\section{Discussion}

$\mathrm{MN}$ is expressed in cells when either acentric chromosome fragments or whole chromosomes fail to be segregated to the daughter nuclei during mitosis ${ }^{9)}$. It also seems that the CBMN assay can be used to measure not only whole chromosome loss or chromosome breaks but also excision-repair events ${ }^{8,22)}$. Previous studies ${ }^{7,17,35)}$ suggested that MN frequency could be one suitable index to evaluate the clastogenic and aneuploid effects in relation to occupational and environmental exposure to VCM. Consistent with previous studies ${ }^{7,15-17)}$, our study suggested that VCM-exposed workers did have a risk of increased $\mathrm{MN}$ frequency that was significantly higher than that of the unexposed control group and that there was a significant dose-response relationship between $\mathrm{MN}$ frequency and VCM exposure.

This study also suggested that smoking status is one of the factors influencing the risk of $\mathrm{MN}$ formation in that $\mathrm{MN}$ frequency in smokers was significantly higher than in nonsmokers within the control group,

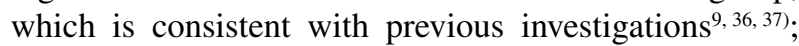
however, there was no significant association between smoking status and MN frequency in the VCM exposure group. A reasonable explanation might be that VCM exposure masked the association between smoking and $\mathrm{MN}$ frequency, while in the control group, without the interaction with VCM exposure, the association became apparent.

This study showed that $\mathrm{MN}$ frequency in female VCM-exposed workers was significantly higher than in male VCM-exposed workers, which is consistent with other evidence ${ }^{38)}$ showing that $\mathrm{MN}$ frequency tended to be greater in females relative to males. The increase in $\mathrm{MN}$ frequency in females can be accounted for by the greater tendency of the $\mathrm{X}$ chromosome to be lost as an $\mathrm{MN}$ relative to other chromosomes and to the fact that females have two copies of the chromosome compared with only one in males ${ }^{39,40)}$. In this study, the fact that the $\mathrm{BMD}_{10}$ value for females was lower than that for males may also be attributable to female VCM-exposed workers being more susceptible than male VCM-exposed workers.

To explore the biologic exposure limit of chromosome damage induced by occupational VCM exposure, the BMD and BMDL of the VCM cumulative exposure dose were calculated as the $10 \%$ response level of chromosome damage using the log-logistic model of BMDS. The present study is the first use of the $\mathrm{BMDL}_{10}$ method to establish VCM occupa- tional exposure safety values in central China. As we mentioned above, the results of $\mathrm{BMD}_{10}$ also suggest that female VCM-exposed workers are more prone to VCM-induced chromosome damage than males. According to the current VCM permissible exposure limit of $1 \mathrm{ppm}\left(2.79 \mathrm{mg} / \mathrm{m}^{3}\right)$ in most developed countries and assuming a typical work duration of $40 \mathrm{yr}$, a worker's VCM cumulative exposure dose would be $3,225 \mathrm{mg}$-year, which is higher than the $\mathrm{BMDL}_{10}$ for all VCM-exposed workers and for male VCM-exposed workers and much higher than the $\mathrm{BMDL}_{10}$ for female VCM-exposed workers in this study. This result is consistent with other biomarker studies of VCM risk assessment. For example, a study of VCM-specific mutant protein biomarkers in male VCM workers in France and Taiwan ${ }^{41)}$ suggested that the current exposure limit was too high to protect against a statistically significant increase in these biomarkers and that an acceptably safe level would be about one quarter of the current level or the equivalent of an approximately $800 \mathrm{mg}$-year cumulative exposure dose for males, which compares favorably to the $670 \mathrm{mg}$-year cumulative exposure dose for males arrived at in this present study. So, although there were uncertainties about our BMD and BMDL estimates, the evidence we have presented is sufficient to warrant further studies on VCM risk of genotoxicity and further scrutiny of the current VCM occupational exposure limits.

This study has several strengths. First, it is an occupational epidemiological study with a relative large sample size. Second, for the first time, the study performed an analysis of the dose-response relationship between VCM cumulative exposure dose and chromosome damage risk using the BMD method in central China and to confirm the previous results in a Shanghai study, and based on this, we derived the VCM-exposed workers' $\mathrm{BMDL}_{10}$, including for males and females, for chromosome damage on the basis of $\mathrm{MN}$ frequency. The population and location of the two studies are different and independent, and the results of both are similar, although the FRs in the present study are not the same as those from the previous study. Likewise, the covariates in this model are not exactly the same as those in the previous study (for example, for Table 1 in the present study, multivariate Poisson regression models were used when these covariates were adjusted by each other), but the two studies can be used as evidence supporting that VCM-exposed workers did have a risk of increased $\mathrm{MN}$ frequency that was significantly higher than the unexposed control group and that there was a strong dose-response relationship between VCM cumulative exposure dose (CED) and the increased risk of a higher MN frequency in the VCM exposure group. The BMD method can be used to evaluate the 
relationship between the genotoxicity indicator and the exposure dose of chemical carcinogens. We did derive the values of BMD and BMDL of the VCM cumulative exposure dose as a chromosome damage indicator in the present study, although they were not the same as those in the previous study; however, we did see that the $\mathrm{BMD}_{10}$ value for females was lower than that for males in both the previous and present study, which suggested that female VCM-exposed workers were more susceptible than male VCM-exposed workers. The estimated values in the two studies can be used to guide policy for how to establish the VCM occupational exposure limits.

In the meantime, our study has several limitations. First, the present study is a case-control study, VCM-exposed workers constituting the cases and controls being school workers who had no exposure, so the study results could be distorted by some bias in that the school workers must be different from the plant workers in many aspects. Second, our study did not directly measure personal VC exposure; instead, exposure assessment was based on job description, work duration, air concentration of VCM at work sites through environmental monitoring and exposure information taken from the published literature. Furthermore, multi-group classification of cumulative exposure introduced additional variation to the exposure assessment. These all contributed to the overarching uncertainty surrounding the dose-response model and the final estimates. Third, unlike hepatic angiosarcoma, MN remains a nonspecific disease marker, and other confounding factors may influence it, such as age, gender, diet, lifestyle factors, disease and genetic factors ${ }^{9}$. For example, our study did not have quantitative data about smoking habits, and in the absence of such data, the simple comparison of smokers versus nonsmokers might be misleading.

In conclusion, VCM exposure was shown to have likely induced chromosome damage in VCM-exposed workers at exposure levels much lower than the current VCM occupational exposure limits, which invites further investigations on a safety level to protect against genotoxicity than the current standard affords. As a biomarker for genotoxicity, chromosome damage data can be used for dose-response assessment and for estimating benchmark doses for the purposes of deriving OELs. Future work is highly desirable to investigate noncarcinogenic health effects induced by VCM and to produce better dose-response data, hence better estimation of BMDs and better quantification of OELs.

Acknowledgment: This work was partly supported by the National Natural Science Foundation of China (30671740), the 973 Program (2011CB503801), the
Shanghai Bureau of Public Health (grant 08GWD12) and NIH grants R01-OH04192 and P30-ES09089.

\section{References}

1) Sherman M. Vinyl chloride and the liver. J Hepatol 2009; 51: 1074-81.[PubMed] [CrossRef]

2) Binetti R, Costamagna FM, Marcello I. Vinyl chloride and 1,2-dichloroethane: classification and assessment of carcinogenicity, guidelines, threshold values, and standards developed by national and international entities, organizations, and agencies. Epidemiol Prev 2001; 25: 31-9.[PubMed] [CrossRef]

3) Giri AK. Genetic toxicology of vinyl chloridea review. Mutat Res 1995; 339: 1-14.[PubMed] [CrossRef]

4) Barbin A, Besson F, Perrard $\mathrm{MH}$, et al. Induction of specific base-pair substitutions in E. coli trpA mutants by chloroethylene oxide, a carcinogenic vinyuhchloride metabolite. Mutat Res 1985; 152: 147-56.[PubMed] [CrossRef]

5) McCann J, Simmon V, Streitwieser D, Ames BN. Mutagenicity of chloroacetaldehyde, a possible metabolic product of 1,2-dichloroethane (ethylene dichloride), chloroethanol (ethylene chlorohydrin), vinyl chloride, and cyclophosphamide. Proc Natl Acad Sci USA 1975; 72: 3190-3.[PubMed] [CrossRef]

6) Fucic A, Horvat D, Dimitrovic B. Mutagenicity of vinyl chloride in man: comparison of chromosome aberrations with micronucleus and sister-chromatid exchange frequencies. Mutat Res 1990; 242: 265-70.[PubMed] [CrossRef]

7) Ji F, Wang W, Xia ZL, et al. Prevalence and persistence of chromosomal damage and susceptible genotypes of metabolic and DNA repair genes in Chinese vinyl chloride-exposed workers. Carcinogenesis 2010; 31: 648-53.[PubMed] [CrossRef]

8) Dhillon VS, Thomas P, Iarmarcovai G, KirschVolders M, Bonassi S, Fenech M. Genetic polymorphisms of genes involved in DNA repair and metabolism influence micronucleus frequencies in human peripheral blood lymphocytes. Mutagenesis 2011; 26: 33-42.[PubMed] [CrossRef]

9) Fenech M, Bonassi S. The effect of age, gender, diet and lifestyle on DNA damage measured using micronucleus frequency in human peripheral blood lymphocytes. Mutagenesis 2011; 26: 43-9.[PubMed] [CrossRef]

10) Cheng J, Leng S, Li H, et al. Suboptimal DNA repair capacity predisposes coke-oven workers to accumulate more chromosomal damages in peripheral lymphocytes. Cancer Epidemiol Biomarkers Prev 2009; 18: 987-93.[PubMed] [CrossRef]

11) Ishikawa $H$, Ishikawa $T$, Miyatsu $Y$, Kurihara K, Fukao A, Yokoyama K. A polymorphism of the methionine synthase reductase gene increases chromosomal damage in peripheral lymphocytes in smokers. Mutat Res 2006; 599: 135-43.[PubMed] [CrossRef]

12) Perez-Cadahia B, Laffon B, Valdiglesias V, Pasaro E, Mendez J. Cytogenetic effects induced by Prestige oil on human populations: the role of polymorphisms in genes involved in metabolism and DNA repair. Mutat Res 2008; 653: 117-23.[PubMed] [CrossRef]

13) Qiu L, Leng S, Wang Z, Dai Y, Zheng Y, Wang Z. Path analysis of biomarkers of exposure and 
early biological effects among coke-oven workers exposed to polycyclic aromatic hydrocarbons. Cancer Epidemiol Biomarkers Prev 2007; 16: 1193-9.[PubMed] [CrossRef]

14) Rohr P, da Silva J, Erdtmann B, Saffi J, Guecheva TN, Antonio Pegas Henriques J, Kvitko K. BER gene polymorphisms (OGG1 Ser326Cys and XRCC1 Arg194Trp) and modulation of DNA damage due to pesticides exposure. Environ Mol Mutagen 2011; 52: 20-7.[PubMed] [CrossRef]

15) Wang W, Qiu YL, Ji F, et al. Genetic polymorphisms in metabolizing enzymes and susceptibility of chromosomal damage induced by vinyl chloride monomer in a Chinese worker population. J Occup Environ Med 2010; 52: 163-8.[PubMed] [CrossRef]

16) Wen-Bin $M$, Wei W, Yu-Lan Q, Fang J, Zhao-Lin $\mathrm{X}$. Micronucleus occurrence related to base excision repair gene polymorphisms in Chinese workers occupationally exposed to vinyl chloride monomer. J Occup Environ Med 2009; 51: 578-85.[PubMed] [CrossRef]

17) Wang Q, Ji F, Sun Y, et al. Genetic polymorphisms of XRCC1, HOGG1 and MGMT and micronucleus occurrence in Chinese vinyl chloride-exposed workers. Carcinogenesis 2010; 31: 1068-73.[PubMed] [CrossRef]

18) Crump KS. A new method for determining allowable daily intakes. Fundamental and Applied Toxicology 1984; 4: 854-71.[PubMed] [CrossRef]

19) Filipsson AF, Sand S, Nilsson J, Victorin K. The benchmark dose method-review of available models, and recommendations for application in health risk assessment. Crit Rev Toxicol 2003; 33: 505-42.[PubMed] [CrossRef]

20) Gaylor D, Ryan L, Krewski D, Zhu YL. Procedures for calculating benchmark doses for health risk assessment. Regulatory Toxicology and Pharmacology 1998; 28: 150-64.[PubMed] [CrossRef]

21) Falk Filipsson A, Victorin K. Comparison of available benchmark dose softwares and models using trichloroethylene as a model substance. Regul Toxicol Pharmacol 2003; 37: 343-55.[PubMed] [CrossRef]

22) Fenech M. the cytokinesis-block micronucleus technique - a detailed description of the method and its application to genotoxicity studies in human-populations. Mutation Research 1993; 285: 35-44.[PubMed] [CrossRef]

23) Fitzgerald DJ, Robinson NI. Development of a tolerable daily intake for $\mathrm{N}$-nitrosodimethylamine using a modified benchmark dose methodology. J Toxicol Environ Health A 2007; 70: 1670-8.[PubMed] [CrossRef]

24) Lei LJ, Chen L, Jin TY, Nordberg M, Chang XL. Estimation of benchmark dose for pancreatic damage in cadmium-exposed smelters. Toxicol Sci 2007; 97: 189-95.[PubMed] [CrossRef]

25) Lin T, Tai-Yi J. Benchmark dose approach for renal dysfunction in workers exposed to lead. Environ Toxicol 2007; 22: 229-33.[PubMed] [CrossRef]

26) Piersma AH, Janer G, Wolterink G, et al. Quantitative extrapolation of in vitro whole embryo culture embryotoxicity data to developmental toxicity in vivo using the benchmark dose approach. Toxicol Sci 2008; 101: 91-100.[PubMed] [CrossRef]

27) Sand S, Victorin K, Filipsson AF. The current state of knowledge on the use of the benchmark dose concept in risk assessment. J Appl Toxicol 2008; 28: 405-21.[PubMed] [CrossRef]

28) Shao B, Jin TY, Wu XW, Kong QH, Ye TT. Application of benchmark dose (BMD) in estimating biological exposure limit (BEL) to cadmium. Biomed Environ Sci 2007; 20: 460-4.[PubMed] [CrossRef]

29) Suwazono $Y$, Nagashima S, Okubo $Y$, et al. Estimation of the number of working hours critical for the development of mental and physical fatigue symptoms in Japanese male workers - application of benchmark dose method. Am J Ind Med 2007; 50: 173-82.[PubMed] [CrossRef]

30) Thomas RS, Allen BC, Nong A, et al. A method to integrate benchmark dose estimates with genomic data to assess the functional effects of chemical exposure. Toxicol Sci 2007; 98: 240-8.[PubMed] [CrossRef]

31) Wang W, Qiu Y, Jiao J, et al. Genotoxicity in vinyl chloride-exposed workers and its implication for occupational exposure limit. Am J Ind Med 2011; 54: 800-10.[PubMed] [CrossRef]

32) Zhu S, Wang A, Xia Z. Polymorphisms of DNA repair gene XPD and DNA damage of workers exposed to vinylchloride monomer. Int $\mathrm{J} \mathrm{Hyg}$ Environ Health 2005; 208: 383-90.[PubMed] [CrossRef]

33) WHO (2000). Chapter 5.16 Vinyl chloride. Air Quality Guidelines - Second Edition.[PubMed] [CrossRef]

34) Fenech $M$. The in vitro micronucleus technique. Mutat Res 2000; 455: 81-95.[PubMed] [CrossRef]

35) Fucic A, Garaj-Vrhovac V, Barkovic D, Kubelka D. The sensitivity of the micronucleus assay for the detection of occupational exposure to vinyl chloride monomer. Mutat Res 1994; 325: 53-6.[PubMed] [CrossRef]

36) Bonassi S, Neri M, Lando C, et al. Effect of smoking habit on the frequency of micronuclei in human lymphocytes: results from the Human MicroNucleus project. Mutat Res 2003; 543: 155-66.[PubMed] [CrossRef]

37) Di Giorgio C, De Meo MP, Laget M, Guiraud H, Botta A, Dumenil G. The micronucleus assay in human lymphocytes: screening for inter-individual variability and application to biomonitoring. Carcinogenesis 1994; 15: 313-7.[PubMed] [CrossRef]

38) Bonassi S, Fenech M, Lando C, et al. HUman MicroNucleus project: international database comparison for results with the cytokinesis-block micronucleus assay in human lymphocytes: I. Effect of laboratory protocol, scoring criteria, and host factors on the frequency of micronuclei. Environ Mol Mutagen 2001; 37: 31-45.[PubMed] [CrossRef]

39) Norppa H, Falck GC. What do human micronuclei contain? Mutagenesis 2003; 18: 221-33.[PubMed] [CrossRef]

40) Tucker JD, Nath J, Hando JC. Activation status of the $\mathrm{X}$ chromosome in human micronucleated lymphocytes. Hum Genet 1996; 97: 471-5.[PubMed] [CrossRef]

41) Brandt-Rauf PW, Luo JC, Cheng TJ, et al. Molecular biomarkers and epidemiologic risk assessment. Hum Ecol Risk Assess 2002; 8: 1295301.[PubMed] [CrossRef] 